\title{
A NOTE ON EXTENSIONS OF SEMIGROUPS OF *-ENDOMORPHISMS
}

\author{
WILLIAM ARVESON AND AKITAKA KISHIMOTO
}

(Communicated by Palle E. T. Jorgensen)

\begin{abstract}
We present a relatively elementary proof that every $E_{0}$-semigroup acting on a type $I_{\infty}$ factor can be extended to a one-parameter group of *-automorphisms acting on a larger type $I_{\infty}$ factor.
\end{abstract}

\section{INTRODUCTION}

An $E_{0}$-semigroup is a semigroup $\alpha=\left\{\alpha_{t}: t \geq 0\right\}$ of normal $*$-endomorphisms of the von Neumann algebra $\mathscr{B}(H)$ of all bounded operators on a Hilbert space $H$, which satisfies $\alpha_{t}(1)=1$ for every $t$ and is weak*-continuous in the natural sense (i.e., $\omega\left(\alpha_{t}(A)\right)$ should be continuous in $t$ for fixed $A \in$ $\mathscr{B}(H)$ and $\left.\omega \in \mathscr{B}(H)_{*}\right)$. A basic result in the theory of $E_{0}$-semigroups asserts that every $E_{0}$-semigroup $\alpha$ can be extended to a one-parameter automorphism group of a larger type $I_{\infty}$ factor, in that there is a Hilbert space $K$ (separable if $H$ is separable) and a one-parameter group $\tilde{\alpha}=\left\{\tilde{\alpha}_{t}: t \in \mathbb{R}\right\}$ of *-automorphisms of $\mathscr{B}(K \otimes H)$ such that

$$
1_{K} \otimes \alpha_{t}(A)=\tilde{\alpha}_{t}\left(1_{K} \otimes A\right),
$$

for every $t \geq 0, A \in \mathscr{B}(H)$. This result was asserted by Powers and Robinson [4], but there is a gap in their argument. Subsequently, the result was established in [2, Corollary 5.21] as a consequence of an analysis of the state space of certain $C^{*}$-algebras. Unfortunately, that proof is very indirect, and a number of individuals have expressed interest in having a proof that is based on more conventional technology. The purpose of this note is to give a proof of the following result, which is based on a minor variation of the F. and M. Riesz theorem for $C^{*}$-dynamical systems [1, Theorem 5.3].

Theorem A. Let $\alpha=\left\{\alpha_{t}: t \geq 0\right\}$ be a weak ${ }^{*}$-continuous semigroup of normal *-endomorphisms of a von Neumann algebra $M$ having separable predual, which satisfies $\alpha_{t}(1)=1$ for every $t \geq 0$. Then there is a nondegenerate normal representation $\pi$ of $M$ on a separable Hilbert space $K$ and a strongly continuous

Received by the editors April 5, 1991.

1980 Mathematics Subject Classification (1985 Revision). Primary 46L40; Secondary 81E05.

Key words and phrases. von Neumann algebras, semigroups, automorphism groups.

This research was supported in part by NSF grant DMS89-12362. 
one-parameter unitary group acting on $K$ such that

$$
\pi \circ \alpha_{t}(x)=U_{t} \pi(x) U_{t}^{*},
$$

for every $x \in M, t \geq 0$.

We remark that the conclusion remains true if one drops the separability hypothes on $M_{*}$, except that of course $K$ need no longer be separable. Theorem A implies the result discussed above in the case where $M$ is a type $I_{\infty}$ factor $\mathscr{B}(H)$, since in that case $\pi$ must be a multiple of the identity representation of $\mathscr{B}(H), \pi(A)=1_{K} \otimes A, A \in \mathscr{B}(H)$.

\section{EXTENDING SEMIGROUPS TO GROUPS}

Let $\alpha=\left\{\alpha_{t}: t \geq 0\right\}$ be a semigroup of isometric *-endomorphisms of a $C^{*}$ algebra $A$. An extension of $\alpha$ is a triple $(B, \beta, \theta)$ consisting of a $C^{*}$-algebra $B$, a one-parameter group $\beta=\left\{\beta_{t}: t \in \mathbb{R}\right\}$ acting on $B$ as $*$-automorphisms, and a faithful $*$-homomorphism $\theta: A \rightarrow B$, which is equivariant in the sense that

$$
\beta_{t} \circ \theta=\theta \circ \alpha_{t}, \quad t \geq 0 .
$$

The extension $(B, \beta, \theta)$ is said to be minimal if $\bigcup_{t \in \mathbb{R}} \beta_{t}(\pi(A))$ is norm-dense in $B$.

Remarks. It is obvious that we can modify any extension $(B, \beta, \theta)$ so as to obtain a smaller extension that is minimal. Moreover, there is a natural notion of (equivariant) isomorphism of extensions of a given $(A, \alpha)$, and any two minimal extensions of $(A, \alpha)$ are isomorphic in this natural sense.

Note too that we have made no assertions about continuity of $\alpha$ or $\beta$ in the time parameter $t$. Indeed, for the application we require, the $C^{*}$-algebraic continuity condition

$$
\lim _{t \rightarrow 0}\left\|\alpha_{t}(a)-a\right\|=0
$$

fails for most $a \in A$. Nevertheless, if $(A, \alpha)$ is continuous in the sense that the above norm limit is 0 for every $a \in A$, then every minimal extension of $(A, \alpha)$ is easily seen to be a $C^{*}$-dynamical system.

There is a construction of extensions of semigroups $(A, \alpha)$ that is similar to more familiar constructions for, say, semigroups of isometries acting on Hilbert spaces. Since we do not know a reference for the result we need, we sketch the argument for completeness.

Proposition 2.1. Every semigroup $(A, \alpha)$ of isometric *-endomorphisms has a minimal extension $(B, \beta, \theta)$.

Proof. Consider the $C^{*}$-algebra $C$ of all bounded $A$-valued functions $F: \mathbb{R} \rightarrow$ $A$ relative to the sup norm

$$
\|F\|=\sup _{x \in \mathbb{R}}\|F(x)\| .
$$

The set $J$ of all functions $F \in C$ satisfying the condition

$$
\lim _{x \rightarrow \infty}\|F(x)\|=0
$$

is a closed ideal in $C$, and we may consider the quotient $C^{*}$-algebra $B=C / J$. Let $F \mapsto \dot{F}$ be the natural projection of $C$ onto $B$. 
The real line acts naturally on $C$ by translations and $J$ is translationinvariant; thus we can define a one-parameter group $\beta=\left\{\beta_{t}: t \in \mathbb{R}\right\}$ of automorphisms of $B$ by $\beta_{t}(\dot{F})=\dot{F}_{t}$, where $F_{t}(x)=F(x+t)$ for $x, t \in \mathbb{R}$. Finally, define a $*$-homomorphism $\theta: A \rightarrow B$ by $\theta(a)=\dot{F}^{a}$ where $F^{a}$ is the element of $B$ defined by

$$
F^{a}(x)= \begin{cases}\alpha_{x}(a), & \text { for } x \geq 0, \\ a, & \text { for } x<0 .\end{cases}
$$

We leave it for the reader to check that $(B, \beta, \theta)$ is an extension of $(A, \alpha)$, and thus one obtains a minimal extension by passing to an appropriate $C^{*}$ subalgebra of $B$.

\section{Proof of Theorem A}

Let $\alpha=\left\{\alpha_{t}: t \geq 0\right\}$ be a weak*-continuous semigroup of unital *-endomorphisms of a von Neumann algebra $M$, and let $(B, \beta, \theta)$ be a minimal extension of $(M, \alpha)$ in the sense of the preceding section. The $C^{*}$-algebra $B$ is in this case the norm-closure of a nested family of von Neumann algebras. More precisely, for every $t \in \mathbb{R}$ we can define a unital $C^{*}$-algebraic embedding $\theta_{t}: M \rightarrow B$ by $\theta_{t}=\beta_{t} \circ \theta$. Thus, we obtain a one-parameter family $\left\{M_{t}: t \in \mathbb{R}\right\}$ of subalgebras of $B$ via $M_{t}=\beta_{t}(M)$.

Each $M_{t}$ is isomorphic to $M$ and can be considered as a von Neumann algebra. The relation $\beta_{s} \circ \theta=\theta \circ \alpha_{s}$ for $s \geq 0$ translates into

$$
\theta_{t+s}=\theta_{t} \circ \alpha_{s}, \quad s \geq 0, t \in \mathbb{R} .
$$

In particular, if $t_{1} \geq t_{2}$ then the formula

$$
\theta_{t_{1}}=\theta_{t_{2}} \circ \alpha_{t_{1}-t_{2}}
$$

implies that $M_{t_{1}} \subseteq M_{t_{2}}$. Moreover, since $\alpha_{t_{1}-t_{2}}$ is a normal *-endomorphism of $M$, the inclusion $M_{t_{1}} \subseteq M_{t_{2}}$ is a normal inclusion of von Neumann algebras. Finally, by minimality of $(B, \beta, \theta)$ it follows that the union

$$
B_{0}=\bigcup_{t>-\infty} M_{t}
$$

is a norm-dense unital *-subalgebra of $B$.

This filtration of $B$ into von Neumann algebras makes it possible to speak of locally normal states of $B$ and locally normal mappings of $B$ into itself. For example, a bounded linear functional $\rho \in B^{*}$ is called locally normal if the restriction of $\rho$ to $M_{t}$ is normal for every $t \in \mathbb{R}$. The locally normal elements of $B^{*}$ are a norm-closed linear subspace of $B^{*}$. Similarly, because every $\alpha_{t}$ is a normal endomorphism of $M$ it follows that for every $t \in \mathbb{R}, \beta_{t}$ is a locally normal $*$-automorphism of $B$ in the sense that $\beta_{t}$ restricts to a normal $*$-homomorphism of $M_{s}$ into $M_{t+s}$ for every $s \in \mathbb{R}$.

We will show that there is a locally normal state $\omega$ on $B$ such that

3.1.a. $\omega \uparrow_{M_{0}}$ is a faithful state of $M_{0}$, and

3.1.b. The representation $\pi: B \rightarrow \mathscr{B}\left(H_{\omega}\right)$ obtained from $\omega$ via the $G N S$ construction is covariant in the sense that there is a strongly continuous oneparameter unitary group acting on $H_{\omega}$ which satisfies

$$
\pi\left(\beta_{t}(x)\right)=U_{t} \pi(x) U_{t}^{*},
$$

for every $x \in B, t \in \mathbb{R}$. 
Notice that the representation $\pi_{0}: M \rightarrow \mathscr{B}\left(H_{\omega}\right)$ given by $\pi_{0}=\pi \circ \theta$ is normal because $\omega \circ \theta$ is a normal state of $M$, it is nondegenerate, and it is faithful because of 3.1.a. The separability of $H_{\omega}$ follows from the fact that if $C$ is a countable set that is strongly dense in the unit ball of $M$ then

$$
\bigcup_{n=1}^{\infty} \pi\left(\beta_{-n} \circ \theta(C)\right) \xi_{\omega},
$$

$\xi_{\omega}$ denoting the canonical cyclic vector associated with $\pi$, is a countable spanning set in $H_{\omega}$. Thus Theorem A follows from the existence of such a state.

In order to exhibit such a locally normal state, we claim first that every normal state of $M_{0}$ can be extended to a locally normal state of $B$. To see that, choose any normal state $\rho_{0}$ of $M_{0}=\theta(M)$. By an obvious induction we can find, for every $n \geq 1$, a normal state $\rho_{n}$ of $M_{-n}$ such that

$$
\rho_{n} \uparrow M_{-n+1}=\rho_{n-1}
$$

for every $n=1,2, \ldots$ (note that here we use normality of the inclusion of $M_{-n+1}$ in $\left.M_{-n}\right)$. Because the family of linear functionals $\left\{\rho_{n}\right\}$ is coherent, we can define a linear functional $\rho_{\infty}$ on $B_{0}$ by

$$
\rho_{\infty}(x)=\lim _{n \rightarrow \infty} \rho_{n}(x),
$$

for $x \in M_{0} \cup M_{-1} \cup M_{-2} \cup \cdots=B_{0}$, and it is obvious that $\rho_{\infty}$ extends by continuity to a (necessarily locally normal) state of $B$.

In particular, if we start with a faithful locally normal state of $M$, as we may because $M$ has separable predual, then we may conclude that there is a locally normal state of $B$ that restricts to a faithful state of $M_{0}$.

Next, we observe that if $\omega$ is any locally normal state of $B$ and $x$ is any element of $B$, then the function $f(t)=\omega\left(\beta_{t}(x)\right)$ is a continuous function of $t \in \mathbb{R}$. It is clearly enough to check this assertion for elements $x$ in the dense subalgebra $B_{0}$, so fix such an $x$ and suppose that $x$ belongs to $M_{\lambda}$. Fix $t_{0} \in \mathbb{R}$. To show that $f$ is continuous at $t_{0}$ we first choose $T>0$ large enough that $T+t_{0}>0$. Because $M_{\lambda}=\beta_{\lambda} \circ \theta(M)$, we may find an element $a \in M$ such that

$$
x=\beta_{\lambda} \circ \theta(a)
$$

and hence for $|h|<T+t_{0}$ we have

$$
\beta_{t_{0}+h}(x)=\beta_{\lambda-T} \circ \beta_{t_{0}+T+h} \circ \theta(a)=\beta_{\lambda-T} \circ \theta\left(\alpha_{t_{0}+T+h}(a)\right)=\theta_{\lambda-T}\left(\alpha_{t_{0}+T+h}(a)\right) .
$$

Hence

$$
f\left(t_{0}+h\right)=\omega\left(\theta_{\lambda-T}\left(\alpha_{t_{0}+T+h}(a)\right)\right.
$$

must tend to $f\left(t_{0}\right)$ as $h \rightarrow 0$ because $\alpha_{t_{0}+T+h}(a)$ tends $\sigma$-weakly to $\alpha_{t_{0}+T}(a)$ and $\omega \circ \theta_{\lambda-T}$ is a normal linear functional on $M$ by local normality of $\omega$.

Now by the preceding paragraphs, we may find a locally normal state $\omega_{0}$ of $B$ that restricts to a faithful state of $\theta(M)$. Let $\phi$ be any positive function in $L^{1}(\mathbb{R})$ whose Fourier transform has compact support, and is normalized so that

$$
\int_{-\infty}^{+\infty} \phi(t) d t=1 .
$$


Define a new state $\omega$ of $B$ by

$$
\omega(x)=\int_{-\infty}^{+\infty} \phi(t) \omega_{0}\left(\beta_{t}(x)\right) d t, \quad x \in B .
$$

We claim that $\omega$ is a locally normal state of $B$ whose restriction to $\theta(M)$ is faithful, and which has compact spectrum in the sense that if $f \in L^{1}(\mathbb{R})$ is any function whose Fourier transform vanishes on the compact support of $\hat{\phi}$ then

$$
\int_{-\infty}^{+\infty} f(t) \omega\left(\beta_{t}(z)\right) d t=0
$$

for every $z \in B$. Indeed, $\omega$ is locally normal because for every $T>0$ the linear functional

$$
\omega_{T}(x)=\int_{-T}^{+T} \phi(t) \omega_{0}\left(\beta_{t}(x)\right) d t
$$

is locally normal (by an obvious application of the bounded convergence theorem), and since

$$
\left\|\omega-\omega_{T}\right\| \leq \int_{-\infty}^{-T} \phi(t) d t+\int_{T}^{\infty} \phi(t) d t
$$

tends to zero as $T \rightarrow \infty$, it follows that $\omega$ is locally normal.

To see that $\omega \uparrow \theta(M)$ is faithful, choose $a \in M$ for which $\omega\left(a^{*} a\right)=0$, i.e.,

$$
\int_{-\infty}^{+\infty} \phi(t) \omega_{0}\left(\beta_{t}\left(\theta\left(a^{*} a\right)\right)\right) d t=0 .
$$

Since $\phi$ is everywhere positive and the integrand is nonnegative and continuous in $t$, it follows that $\omega_{0}\left(\beta_{t}\left(\theta\left(a^{*} a\right)\right)\right)$ vanishes for all real $t$. Hence it vanishes in particular for $t=0$, which implies that $a=0$ because $\omega_{0} \circ \theta$ is faithful on $M$. We may conclude that $\omega$ has property 3.1.a.

Finally, property 3.2 follows from the fact that if $f$ is any integrable function on $\mathbb{R}$ whose Fourier transform vanishes on the support of $\hat{\phi}$, then $(f * \phi)^{-}=$ $\hat{f} \hat{\phi}=0$, hence $f * \phi=0$. Thus for every $z \in B$ we have the desired relation

$$
\begin{aligned}
\int_{-\infty}^{+\infty} f(t) \omega\left(\beta_{t}(z)\right) d t & =\int_{-\infty}^{+\infty} \int_{-\infty}^{+\infty} f(t) \phi(s) \omega\left(\beta_{t+s}(z)\right) d t d s \\
& =\int_{-\infty}^{+\infty} f * \phi(u) \omega\left(\beta_{u}(z)\right) d u=0
\end{aligned}
$$

The preceding discussion implies that the spectrum of the state $\omega$ relative to the one-parameter automorphism group $\beta$ is compact. In particular, there is a $\lambda \in \widehat{\mathbb{R}}$ for which the spectrum of $\omega$ is contained in the half-line $[\lambda,+\infty)$. At this point, we are in a setting very close to that of the F. and M. Riesz theorem for $C^{*}$-dynamical systems [1, Theorem 5.3], whose conclusion is precisely the relation 3.1.b that we seek. However, there are two ways in which our setting fails to meet exactly the hypotheses of that theorem.

First, Theorem 5.3 of [1] requires that $\lambda$ be 0 or at least nonnegative, whereas since $\omega$ is a state its spectrum is symmetric about the origin in $\widehat{\mathbb{R}}$, and hence the $\lambda$ that we have is surely negative. Second, $(B, \mathbb{R}, \beta)$ is not a $C^{*}$-dynamical system but rather a locally normal automoprhism group acting 
on a $C^{*}$-algebraic inductive limit of von Neumann algebras. The fact that $\lambda$ is negative poses no difficulty; indeed the proof of [1, Theorem 5.3] carries over intact to the case where the spectrum of $\omega$ is contained in any proper half-line of the form $[\lambda,+\infty)$. The fact that $(B, \mathbb{R}, \beta)$ is not a $C^{*}$-dynamical system poses no difficulties either, since the essential thing here is that the group $\beta=\left\{\beta_{t}\right\}$ should be integrable in the sense that for every $x \in B$ and every $f \in L^{1}(\mathbb{R})$, there should exist an element $y \in B$ such that

$$
\rho(y)=\int_{-\infty}^{+\infty} f(t) \rho\left(\beta_{t}(x)\right) d t
$$

for every locally normal $\rho$ in $B^{*}$ [3, p. 300]. The existence of such weak integrals

$$
y=\int_{-\infty}^{+\infty} f(t) \beta_{t}(x) d t
$$

is easily established in the present context using familiar techniques (cf. [1] for example).

\section{REFERENCES}

1. W. Arveson, On groups of automorphisms of operator algebras, J. Funct. Anal 15 (1974), 217-243.

2. $\ldots$ Continuous analogues of Fock space IV: essential states, Acta Math. 164 (1990), 265-300.

3. G. K. Pederson, $C^{*}$-algebras and their automorphism groups, Academic Press, London, 1979.

4. R. T. Powers. and D. Robinson, An index for continuous semigroups of *-endomorphisms of $\mathscr{B}(H)$, J. Funct. Anal. 84 (1989), 85-96.

Department of Mathematics, University of California, Berkeley, California 94705

Department of Mathematics, Hokkaido University, Kita 10 Nishi 8, Kita-ku, Sapporo 060, JAPAN 